A N N A L E S Annales de Bretagne et des Pays de l'Ouest

Anjou. Maine. Poitou-Charente. Touraine

119-3 | 2012

Les abbayes martiniennes

\title{
Les réseaux martiniens en Irlande médiévale
}

Martinian networks in early medieval Ireland

Jean-Michel Picard

\section{(2) OpenEdition}

Journals

Édition électronique

URL : http://journals.openedition.org/abpo/2473

DOI : $10.4000 / a b p o .2473$

ISBN : 978-2-7535-2136-0

ISSN : 2108-6443

Éditeur

Presses universitaires de Rennes

Édition imprimée

Date de publication : 30 octobre 2012

Pagination : 41-54

ISBN : 978-2-7535-2134-6

ISSN : 0399-0826

Référence électronique

Jean-Michel Picard, « Les réseaux martiniens en Irlande médiévale », Annales de Bretagne et des Pays de l'Ouest [En ligne], 119-3 | 2012, mis en ligne le 30 octobre 2014, consulté le 01 mai 2019. URL http://journals.openedition.org/abpo/2473; DOI : 10.4000/abpo.2473 


\title{
Les réseaux martiniens en Irlande médiévale
}

\author{
Jean-Michel PICARD \\ Professeur en Études Médiévales, University College Dublin
}

Dans sa Vita Columbani écrite vers 640, Jonas de Bobbio relate le passage de Colomban et de ses compagnons irlandais par Tours en route pour Nantes après son expulsion de Luxeuil par le roi Thierry II de Bourgogne en 610 :

\begin{abstract}
Navigantes ergo per Ligerim ad Turonensem perveniunt urbem, ubi vir sanctus custodes precatur ut scapham porto appropinquent, seque ad sepulcrum B. Martini ire sinant. Quod abnuentes custodes, navigare veloci conatu compellunt, remigeros urgent ut omni virtute cursu celeri portum transeant, gubernatori imperant ut medio fluminis alveo scapham teneat. [...] Mirum in modum medio e fluviolo ad portum pennigero ceu volatu pervenit, portuque recepto viro Dei viam pandit. Ille vero gratias Regi offert aeterno, qui suis sic obtemperare non dedignatur servis. Egressus sane ad sepulcrum B. Martini accedit, et tota ibi nocte in oratione excubat*
\end{abstract}

*Jonas, Vita Columbani, i, 22 (éd. krusch, Bruno Ionae vitae sanctorum Columbani, Vedastis Johannis, Hanovre, Hahn, 1905).
Naviguant donc sur la Loire, ils arrivèrent à la ville de Tours, où l'homme saint pria ses gardiens d'approcher le bateau du port et de le laisser aller au tombeau du bienheureux Martin. Les gardiens refusèrent cette demande et s'empressèrent de naviguer à toute vitesse, pressant les rameurs d'employer toute leur force à doubler le port en cadence rapide et ordonnant au pilote de maintenir le bateau au milieu du lit du fleuve. [...] Chose merveilleuse, le navire parvient du milieu du fleuve au port comme s'il volait sur des ailes et ayant abordé au port, il ouvre la voie à l'homme de Dieu. Quant à lui, il rendit grâces au Roi éternel, qui ne dédaigne pas d'obéir ainsi à ses serviteurs. Sorti du bateau, il se rendit assurément sur la tombe du bienheureux Martin et veilla là toute la nuit en prière.

Le lendemain matin, Colomban est invité à déjeuner par l'évêque Lupare, mais de retour sur le bateau, il apprend que l'or et les provisions des Irlandais ont été volés pendant la nuit. Il invoque alors Martin pour lui demander de réparer cette injustice et le voleur revient spontanément rendre l'or des moines. Réapprovisionnés par l'évêque Lupare, les Irlandais reprennent ensuite leur voyage vers Nantes.

Ce texte est le premier témoignage écrit de la vénération d'un groupe d'Irlandais pour saint Martin. L'hagiographe insiste sur l'importance de cette visite 
au tombeau du saint, qui a lieu contre la volonté des hommes de confiance de Thierry II, craignant probablement les sympathies austrasiennes dans la ville et la possibilité pour Colomban d'échapper à leur contrôle. La facilité avec laquelle Colomban circule dans Tours et l'accueil qu'il reçoit de l'évêque laissent supposer des contacts avec les réseaux irlandais, qu'il faut peut-être placer dans le contexte de rapports commerciaux entre l'Irlande et la vallée de la Loire à la fin du $\mathrm{VI}^{\mathrm{e}}$ siècle $^{1}$. Ces rapports semblent avoir été réguliers. Par exemple, quand Colomban arrive à Nantes, les autorités locales n'ont aucune peine à lui trouver sans délai un navire marchand pour le ramener dans son pays. Les marchandises que les dames de Nantes donnent aux moines irlandais indiquent le genre de denrées qu'exportait la vallée de la Loire : Procula fournit cent muids de vin, deux cents de blé et cent de céréales à faire la bière et Doda lui fait envoyer deux cents muids de blé et cent de méteil ${ }^{2}$. En échange, les Irlandais exportaient des peaux, des manteaux de laine, des chiens et des esclaves. Outre la Vita Columbani, où la présence d'un navire irlandais à Nantes est présentée comme un fait ordinaire, nous avons le témoignage de la Vita Filiberti, où l'on voit les moines de Noirmoutier se ravitailler en vêtements et chaussures auprès de marchands irlandais ${ }^{3}$.

Le témoignage de Jonas de Bobbio concernant l'importance de Martin chez les moines de Bangor est confirmé par Colomban lui-même dans la lettre qu'il écrit aux évêques du Concile de Chalon en 603 :

Sed confiteor conscientiae meae secreta, quod plus credo traditioni patriae meae iuxta doctrinam et calculum octoginta quattuor annorum et Anatolium ab Eusebio ecclesiasticae historiae auctore episcopo et sancto catalogi scriptore Hieronymo laudatum Pascha celebrare, quam iuxta Uictorium nuper dubie scribentem et, ubi necesse erat, nihil definientem, ut ipse in suo testatus prologo, qui post tempora domni Martini et domni Hieronymi et papae Damasi per centum et tres annos sub Hilaro scripsit*.

*Colomban, Lettre ii au Concile de Châlon (éd. Walker, George Stuart, Sancti Columbani Opera, Dublin, DIAS, 1970, p. 12-22).
Mais je confesse du plus profond de ma conscience que, pour la célébration de Pâques, je crois plus à la tradition de ma patrie en accord avec la doctrine et le calcul de 84 ans et avec Anatole, loué par l'évêque Eusèbe, auteur de l'Histoire Ecclésiastique, et par Jérôme, le saint auteur du Catalogue, qu'à celle que suit Victor, qui écrit récemment et de façon douteuse sans rien définir quand c'est nécessaire, comme il l'atteste lui-même dans son prologue et qui, après l'époque des saints Martin et Jérôme, écrivit sous Hilaire un comput couvrant encore 103 ans.

1. Voir la bibliographie sur la question donnée par LEBECQ, Stéphane, « La Neustrie et la mer " dans ATSMA, Hartmut, La Neustrie, 2 vols, Sigmaringen, Jan Thorbecke Verlag, 1989, vol. 1 , p. $405-440$ : p. 412 , n. 36.

2. Jonas, Vita Columbani, I, $22:$ «[...] centum modia esse uini ducentaque frumenti sed et braces centum idemque modia", et plus loin "Aliaque simul nobilis et religiosa femina Doda nomine direxit ducenta frumenti ac promiscue centum modia".

3. Vita Filiberti, 42 (éd. LEVISON, Wilhelm, Monumenta Germaniae Historica, Scriptores Rerum Merovingicarum, 5, Hanovre, Hahn, 1910, p. 583-604) : "Una dierum sedens cum fratribus spiritu prophetice repletus ait eis : "Viri fratres, Deo gratias agamus per omnia quia cito nobis a partibus marinis aduenient oportuna conpendia." Nec multum post Scothorum nauis diversis mercimoniis plena ad litus adfuit qui calciamenta ac uestimenta fratribus larga copia ministrauit. " 
Dans le conflit qui l'oppose aux évêques gaulois, Colomban défend ainsi l'orthodoxie et la précédence des usages irlandais, en invoquant les noms de Jérôme et de Martin contre la tradition plus récente de Victor d'Aquitaine.

La vénération de Colomban pour saint Martin reflète les traditions de la communauté irlandaise dont il était issu. Le rayonnement de Colomban au sein des élites de la Gaule franque et son influence sur les grands fondateurs de monastères que furent saint Amand, Saint Ouen ou saint Philibert ne doivent pas nous faire oublier la filière en amont et son éducation à Bangor sur la côte nord-est de l'Irlande ${ }^{4}$. Bangor fut fondé en 555 par Comgall, disciple du grand Columba (Colum Cille en gaélique), apôtres des Pictes et fondateur de la puissante organisation monastique d'Iona. Bien attestée dans l'hagiographie irlandaise, la tradition des liens étroits entre Bangor et Iona et de la filiation Columba-Comgall-Colomban était encore connue sur le continent au $\mathrm{IX}^{\mathrm{e}}$ siècle $^{5}$. Comme le souligne Jonas de Bobbio au tout début de la Vita Columbani, le véritable nom de Colomban est Columba, et c'est ainsi que le saint lui-même se nomme dans les lettres qu'il écrit à ses moines ou à différents Papes. Le choix de ce nom n'est certainement pas un hasard dans le contexte que nous venons d'évoquer.

La plupart des informations que nous avons sur Columba (= Colum Cille) et le monastère d'Iona nous vient de la Vita Columbae, une grande oeuvre hagiographique en trois livres composée par Adomnán, abbé d'Iona entre 679 et 704 . Occurrence rare pour le haut Moyen Âge irlandais, l'historien moderne a la chance de pouvoir utiliser une oeuvre, écrite peu avant 700, par un érudit qui s'intéresse à l'histoire et à la géographie ecclésiastiques, responsable de la rédaction des Annales d'Iona, auteur d'un $D e$ Locis Sanctis qui sera copié par Bède, et qui, de surplus, nous est parvenue dans un manuscrit rédigé avant 713. C'est le document le plus fiable que nous ayons sur la communauté monastique d'Iona pour les années 570-690 et c'est par lui que nous apprenons que Martin était vénéré à Iona du vivant même de Columba. Au livre III, qui traite des visions et des apparitions angéliques, Adomnán raconte comment Columba contempla la vision de l'âme de l'évêque Colmán emporté au paradis par les anges le jour même de sa mort. Pour marquer l'événement, Columba arrête le travail des moines, déclare un jour férié et leur demande d'inclure le nom de Colmán dans une litanie qui commémorait habituellement celui de saint Martin.

4. Le réseau des monastères du nord de l'Irlande : Iona, Bangor et Derry.

5. Sur cette tradition rapportée par Notker le Bègue, moine de saint Gall, voir PICARD, Jean-Michel, "Schaffhausen Generalia I and the textual transmission of Adomnán's Vita Columbae on the continent ", dans Ní CHATHÁIN, Proinseas et RichTER, Michael (dir.), Ireland and Europe in the early Middle Ages : Texts and transmission, Dublin, Four Courts Press, 2001, p. 95-102. 
[...] iuxta sancti iusionem eadem otiantur die, praeparatisque sacris ad eclesiam ministeriis quasi die sollempni albati cum sancto pergunt. Sed forte, dum inter talia cum modolatione officia illa consueta decantaretur deprecatio in qua sancti Martini commemoratur nomen, subito sanctus ad cantatores eiusdem onomatis ad locum peruenientes: "Hodie, ait, "pro sancto Columbano episcopo" decantare debetis*. ”

*Adomnán, Vita Columbae, 3, 12 (éd. Anderson, Alan Orr et Anderson, Marjorie Ogilvie, Adomnan's Life of Columba, Oxford, Oxford University Press, 19912).
Et selon l'ordre du saint (= Columba), ce jour fut un jour de repos et, ayant préparé la célébration de la messe, ils se rendirent à l'église tout habillés de blanc comme pour une fête solennelle. Mais il arriva que, au cours de l'office, comme ils chantaient mélodieusement la prière habituelle dans laquelle on commémore le nom de saint Martin, le saint dit soudain aux chanteurs qui venaient d'arriver au passage où l'on prononce son nom : "Aujourd'hui vous devez chanter "pour saint Colmán, évêque". "

Le sens de ce passage doit se comprendre dans le contexte du rituel de la messe célébrée à Iona, probablement similaire à celui du missel de Lorrha, qui nous est parvenu dans un manuscrit de la fin du $\mathrm{VIII}^{\mathrm{e}}$ ou début du IX ${ }^{\mathrm{e}}$ siècle. Aux folios 16 et 17 se trouve une litanie intitulée Deprecatio sancti Martini pro populo "Prière de saint Martin pour le peuple " (voir figure 1 et transcription en note 9 infra). Chantée entre l'Épître et l'Évangile, cette litanie ancienne (comme le suggèrent les références aux empereurs et à l'armée romaine ${ }^{6}$ ) demande la protection du seigneur pour l'Église catholique et les diverses catégories de personnes qui la composent. L'épisode de Vita Columbae et la référence à une Deprecatio sancti Martini nous laisse entendre qu'aux $\mathrm{VI}^{\mathrm{e}}$ et $\mathrm{VII}^{\mathrm{e}}$ siècles, les moines d'Iona chantaient une litanie liée à la tradition martinienne et commémoraient ainsi, chaque fois qu'une messe était célébrée, le nom du saint qu'ils considéraient comme une inspiration majeure de leur mouvement. En effet, dans sa présentation du mouvement monastique fondé par Columba, Adomnán insiste sur l'aspect militaire de la vie monastique. Celle-ci est présentée comme " une carrière militaire " (christiana militia) où l'on entre à l'issue d'un " apprentissage des armes " (tirocinium). Les moines d'Iona sont des "soldats du Christ " (Christi milites, Christi commilitones). L'aspect militaire du monachisme martinien est bien connu et a fait l'objet de nombreuses analyses modernes. Le modèle martinien est présent dans la Vita Columbae non seulement au niveau des idéaux, mais aussi au niveau de la forme. La structure même de la Vita est modelée sur celle de la Vita Martini de Sulpice Sévère, avec une double préface

6. Manuscrit Dublin, Royal Irish Academy, D ii 3 (fin VIII ${ }^{\mathrm{e}}$ siècle), $\mathrm{f}^{\circ} 16$ : " Deprecatio sancti Martini pro populo incipit amen Deo gratias//Dicamus omnes : Domine, exaudi et misserere, Domine, misserere//Ex toto cordo et ex tota mente : Qui respices//super terram et facis eam tremere! Oramus!//Pro altissima pace et trancillitate temporum nostrorum, pro sancta aeclessia catholica quae est a finibus//usque ad terminos orbis terrae! Oramus!// Pro pastore N. episcopo et omnibus episcopis et//praespeteris et diaconis et omni clero! Oramus!//Pro hoc loco et inhabitantibus in eo, pro pissimis imperatoribus et omni romano exercitu! Oramus!" 
et une division en trois parties ${ }^{7}$. Les emprunts verbaux confirment la volonté d'identifier Columba à Martin, en particulier dans les passages stratégiques que sont les préfaces et le chapitre final :

Adomnán, Vita Columbae (PRAEF. 1) Beati nostri patroni Christo sufragante uitam discripturus, fratrum flagitationibus obsecundare uolens, in primis eandem lec. turos quosque ammonere procurabo ut fidem dictis adhibeant conpertis et res magis quam uerba perpendant, quae ut estimo inculta et uilia esse uidentur. Meminerintque regnum Dei non in eloquentiae exuberantia sed in fidei flo rulentia constare.
Sulpice Sévère, Vita Martini (I,9) Obsecro autem eos qui lecturi sunt, ut fidem dictis adhibeant, neque me quicquam nisi conpertum et probatum scripsisse arbitrentur; alioquin tacere quam falsa dicere maluissem. (PRAEF. 3-4) Quod si acciderit et $a b$ aliquibus eum legi uideris, bona uenia id a lectoribus postulabis, ut res potius quam uerba perpendant, et aequo animo ferant si aures eorum uiliosus forsitan sermo perculerit. [...] quia regnum Dei non in eloquentia, sed in fide constat. Meminerint etiam salutem saeculo non ab oratoribus, cum utique, si utile fuisset $[\ldots]$

Le nom d'Adomnán est aussi lié à l'un des plus anciens hymnes irlandais en l'honneur de Martin. Il s'agit de l'hymne Martine te deprecor, composé à la fin du VII ${ }^{\mathrm{e}}$ siècle par Óengus mac Tipraite, moine de Clonfad (voir figure 2) ${ }^{8}$. Clonfad avait été fondé par saint Finnian de Clonard $\mathrm{au} \mathrm{VI}^{\mathrm{e}}$ siècle et, selon le Martyrologe d'Óengus, Columba aurait été ordonné prêtre par Etchen, évêque de Clonfad, dans les années 540. L'auteur de la préface bilingue de l'hymne place les circonstances de sa rédaction dans le contexte du circuit de visitation de l'abbé d'Iona en Irlande. Óengus aurait été convoqué au jour de la fête de saint Martin pour répondre d'une accusation, et terrifié à l'idée du châtiment probable, il composa au cours de la nuit précédente un hymne à saint Martin pour se protéger. Quand il se présenta à Adomnán avec son hymne, celui-ci vit en apparition saint Martin qui se tenait à la droite d'Óengus :

7. PICARD, Jean-Michel, "Structural patterns in early Hiberno-Latin hagiography ", Peritia, $\mathrm{n}^{\circ} 4,1985$, p. 67-82.

8. Manuscrit University College Dublin, Franciscan MS A2 (XI ${ }^{\mathrm{e}}$ siècle) p. 24 : "Martine te deprecor pro me rogaris patrem//Christum ac spiritum sanctum habentem Mariam matrem.//Martinus mirus more ore laudauit Deum//puro corde cantauit atque amauit eum.//Electus Dei uiui signa sibi salutis//donauit Deus pacis magnae atque uirtutis.// Verbum Dei locutus secutus in mandatis//uirtutibus impletis mortuis resuscitatis.// Sanans homines lepra cura duplice mira//magnitudine mala egretudine dira.//Deum dominum nostrum passum pro nobis mire//uoluntarie propter nos deprecare Martine./ Martine. " 
Atracht tra Adamnan reme et honorificauit eum cum osculo et omnes mirabantur causam honoris et dixit Adamnan ut uidit Martinum secum conid ar Martan do bith immalle fris dorat honor do. Rosoerad tra amlaid sein Óengus et ostendit hymnum suum et laudauit Adamnan ymnum et dixit gnúis airmitiu for intí gebas ic dul dochum dala no airechta ocus comad dítiu ar cech ngalar ocus nem ar a gabail fo lige fo eirge.
Alors Adomnán se leva devant lui et l'honora en l'embrassant et tous s'étonnèrent de la cause de cet honneur. Et Adomnán dit qu'il avait vu Martin avec lui et que c'est parce que Martin se trouvait avec lui qu'il lui avait rendu honneur. Et alors Óengus fut acquitté et il montra son hymne et Adomnán loua l'hymne et dit : «Honneur personnel à celui qui le récitera en se rendant à une assemblée ou une cour de justice ; il sera aussi une protection contre toute maladie et celui qui le récitera au coucher et au lever ira au ciel. »

Écrite plus de deux cents ans après l'hymne, cette préface utilise un original latin plus ancien et reflète des croyances attestées aux $\mathrm{VII}^{\mathrm{e}}$ et $\mathrm{VIII}^{\mathrm{e}}$ siècles. Le contexte juridique qui y est évoqué trouve un écho dans un autre texte émanant du réseau d'Iona, la Collectio Canonum Hibernensis. Compilé avant 725 par Cú Chuimne d'Iona et Ruben de Dair-Inis ${ }^{9}$, cet important ouvrage représente la somme des canons irlandais rédigés au cours des siècles précédents. La plupart de ces textes s'appuient sur l'autorité de la Bible et des Pères de l'Église. Martin y est considéré comme l'égal de Jérôme, Augustin, Hilaire ou Origène. Ainsi au livre II, c'est lui qui sert d'autorité pour justifier le canon qui autorise le prêtre à accepter tout financement quelle que soit son origine, dans la mesure où cet argent sera utilisé pour des oeuvres charitables et non pour son usage personnel. Identifiant Martin comme autorité juridique par la formule traditionnelle Martinus dicit, le législateur irlandais utilise en fait un épisode des Dialogues de Sulpice Sévère où Martin accepte sans les accepter les cent livres d'argent du viguier Lycontius, car il s'en sert pour libérer des captifs et non pour les besoins de son monastère ${ }^{10}$. Dans le Corpus Iuris Hibernici, Martin est invoqué dans les formules de serment, en association avec l'archange Michel, justicier et peseur des âmes au jugement dernier (formule dar Martain \& dar Michel).

C'est au sein de ce réseau d'Irlande du Nord qu'a été composé un autre hymne en l'honneur de Martin, l'hymne Deus Domine meus, longtemps attribué au Pseudo-Bède, mais correctement identifié par Michael Lapidge comme une oeuvre hiberno-latine du $\mathrm{VII}^{\mathrm{e}}$ siècle. Les caractéristiques métriques de ce poème sont rares et se retrouvent dans l'hymne Martine te deprecor, dans le poème sur la règle du monastère de Bangor, intitulé

9. JASKI, Bart, " $\mathrm{Cu}$ Chuimne, Ruben and the compilation of the Collectio canonum hibernensis ", Peritia, n 14, 2000, p. 51-69.

10. Collectio Canonum Hibernensis (éd. Wasserschleben, Hermann, Die irische Kanonensammlung, Leipzig, Bernard Tauchnitz, 1885) II, 24 : "De eo quod dona iniquorum a sacerdote recipienda sint, ut tamen pauperibus erogentur et captiuis // Martinus dicit: Mox ad eum Liguntius, diuina expertus beneficia peruolauit, $C$ etiam argenti libras obtulit, quas uir beatus nec respuit nec recepit, sed priusquam pondus illud monasterii limen attingeret, redimendis id captiuis deputauit; et cum ei suggereretur, ut aliquid ab eo in sumtum monasterii seruaret, omnibus angustum esse uictum, multis deesse uestimentum, nos, inquit, ecclesia pascat et uestiat ". Cf. Sulpice Sévère, Dialogues, 3, 14. 
Benchuir bona regula, et dans le poème Mundus iste transibit, qui est probablement une œuvre de Colomban ${ }^{11}$. Outre le thème général de demande de protection contre la maladie, la mort et les peines de l'Enfer, présents dans l'hymne Martine te deprecor, l'un des thèmes du Deus Domine meus est celui du pèlerinage à Tours, entreprise difficile comprenant les risques de la traversée en mer et pour laquelle l'aide du Christ et de Martin est sollicitée :

3. Rogo nunc regem regum qui est lumen diuinumut ualeam nunc sanctum uisitare Martinum

4. Christe Deus deorumcuius est numen mirum fac me lugere sanum iuxta sanctum Martinum

6. Mihi adiutoriumerit per naufragium Christi militis miri suffragium Martini

7. Volo te uisitare fac me ad te uenire qui es uirtutis tantae o mi sancte Martine
Je supplie maintenant le Roi des Rois, qui est la lumière divine, qu'il me donne la force d'aller rendre visite à saint Martin. Christ, Dieu des dieux, dont la puissance est miraculeuse, amène-moi saint et sauf pleurer auprès de saint Martin.

Mon secours contre le naufrage sera la faveur de Martin, merveilleux soldat du Christ.

Je veux te rendre visite, fais-moi venir à toi, qui as un tel pouvoir miraculeux, $\mathrm{O}$ mon saint Martin.

Nous reviendrons sur ce thème du pèlerinage à Tours dans le contexte de l'hagiographie irlandaise des $\mathrm{VIII}^{\mathrm{e}}$ et $\mathrm{IX}^{\mathrm{e}}$ siècles.

Si le culte de Martin est particulièrement fort dans les milieux associés au réseau d'Iona, cette communauté ne détient pas le monopole de sa vénération et l'héritage martinien semble avoir fait l'objet d'une rivalité avec la communauté d'Armagh, siège des successeurs de saint Patrick. Nous ne savons pas quand le culte de Martin fut introduit à Armagh. Ce que nous savons, c'est qu'Armagh est non seulement un siège épiscopal, mais aussi le centre d'une importante organisation monastique et que le monachisme se développe dans ce milieu dès l'époque de saint Patrick dans la seconde moitié du $\mathrm{v}^{\mathrm{e}}$ siècle. Dans sa Confession, écrite dans les années 470, Patrick nous parle des moines et moniales (monachi et uirgines) issus des élites irlandaises et participant à sa mission ${ }^{12}$. Nous ne savons pas non plus quand Armagh acquiert les documents martiniens copiés dans le Livre d'Armagh (IX ${ }^{\mathrm{e}}$ siècle). Ce que nous savons c'est que la version de la Vita Martini et des Dialogues de Sulpice Sévère copiée par Ferdomnagh dans le Livre d'Armagh peu avant 807 est une version ancienne qui préserve la division originale des livres de Sulpice ${ }^{13}$. Alors que les manuscrits carolingiens continentaux divisent les Dialogues en trois sections, le Livre d'Armagh préserve la division originale en deux dialogues clairement

11. Texte édité et commenté par LAPIDGE, Michael, « A New Hiberno-Latin hymn on St Martin ", Celtica, n 21, 1990, p. 240-251.

12. Patrick, Confession, (éd. Hanson, Richard Patrick Crosland, et Blanc, Cécile, Saint Patrick: Confession et lettre à Coroticus, Sources Chrétiennes 249, Paris, Éditions du Cerf, 1978) $§ 41$ : «Vnde autem Hiberione qui numquam notitiam Dei habuerunt nisi idola et inmunda usque nunc semper coluerunt quo modo nuper perfecta est plebs Domini et filii Dei nuncupantur, filii Scottorum et filiae regulorum monachi et uirgines Christi esse uidentur".

13. Voir FonTAine, Jacques, Sulpice Sévère. Gallus, Dialogues sur les “ Vertus » de Saint Martin, Sources Chrétiennes 510, Paris, Éditions du Cerf, 2006, p. 19-20. 
identifiés comme ceux de Postumianus et de Gallus ${ }^{14}$. C'est la division du Veronensis, qui date du $\mathrm{VI}^{\mathrm{e}}$ siècle, et il n'est pas impossible qu'Armagh ait cherché à obtenir les oeuvres de Sulpice dès cette époque, qui est celle de son expansion, caractérisée par l'acquisition de reliques romaines et la production de documents proclamant sa suprématie sur toutes les Églises d'Irlande. Bien que le nom de Martin ne soit pas mentionné dans la Vita Patricii écrite par Muirchú vers 690, l'un des chapitres du livre II pourrait être inspiré de la Vita Martini. C'est l'épisode de la tombe du faux chrétien, ou du faux martyr, honorée à tort par les fidèles de la région. Mais contrairement à la Vita Columbae d'Adomnán, il n'y a pas d'emprunts verbaux et la structure du récit est suffisamment différente pour nous laisser supposer une transmission orale plutôt qu'une lecture assidue de Sulpice Sévère ${ }^{15}$. Il faut attendre la fin du VIII ${ }^{\mathrm{e}}$ siècle pour que les hagiographes irlandais expriment clairement une volonté d'associer Martin à Patrick, en introduisant la tradition du passage de Patrick à Tours. La Vita Tertia Patricii, composée à une date indéterminée entre le $\mathrm{IX}^{\mathrm{e}}$ et le $\mathrm{XI}^{\mathrm{e}}$ siècle, fait résider Patrick à Auxerre pendant 40 ans auprès de saint Germain, puis quarante jours à Tours auprès de saint Martin avant son départ pour Lérins ${ }^{16}$. La Vita Tripartita Patricii, oeuvre bilingue du $\mathrm{IX}^{\mathrm{e}}$ siècle, contient les mêmes données - études à Auxerre et séjour à Tours, où Patrick reçoit la tonsure de Martin - mais l'hagiographe va plus loin en faisant de Martin un parent de Patrick. Le livre premier de la Vita Tripartita commence ainsi :

Patraic didiu, di Bretnaib Ail Cluaide a bunadus. Calpurnd ainm a athar, huasalsacart hé. Fótid ainm a senathar, deochon atacomnaic. Concess ainm a mathar, di Francaib di 7 siur do Mártan hí*

*Vita Tripartita Patricii, I, 1, éd. Mulchrone, Kathleen, Bethu Phátraic. The Tripartite Life of Patrick, Dublin, Royal Irish Academy, 1939, p. 5.
Patrick donc était originaire du pays des Bretons de Strathclyde. Son père se nommait Calpurnius et était archiprêtre. Son grand-père se nommait Potitus et avait été diacre. Sa mère se nommait Concessa, originaire du pays des Francs et soeur de Martin.

Ces données biographiques n'ont rien d'historique, mais attestent de l'importance de Tours et de Martin dans les réseaux patriciens de l'époque carolingienne. Les voyages fictifs de Patrick à Rome, à Auxerre, à Tours et

14. Dublin, Trinity College, MS 52 (Book of Armagh) $\mathrm{f}^{\circ} 201 \mathrm{v}^{\circ}:$ " Explicit liber primus Sulpicii Seueri de uita sancti Martini episcopi ; incipit II Postumiani de uirtutibus monachorum "; $\mathrm{f}^{\circ} 215 \mathrm{r}^{\circ}$ : " Explicit secundus liber; incipit tertius Galli ».

15. Muirchú, Vita Patricii, II, 2 (éd. BIELER, Ludwig, The Patrician Texts in the Book of Armagh, Dublin, D.I.A.S., 1979, p. 114), cf. Sulpice Sévère, Vita Martini, c. 11 (éd. FonTAINE, Jacques, Sulpice Sévère, Vie de Saint Martin, Sources Chrétiennes 133, Paris, Éditions du Cerf, 1967, p. 276).

16. Vita Tertia Patricii (éd. BIELER, Ludwig, Four Latin Lives of St. Patrick, Dublin, D.I.A.S., 1971, p. 118-190) $§ 22$ : "Tunc sanctus Patricius cogitauit per angelicam uisionem exire, ut sanctas scripturas disceret, ut posset eos docere. Perrexit ergo ad regiones Gallorum et uenit ad sanctum episcopum Germanum, uirum sapientissimum et honoratum ab omnibus Gallis principem Altisiodori ciuitatis, et mansit apud eum quadraginta annis legens et implens diuinas scripturas, et uirgo corpore et spiritu. Et postea uenit ad Martinum et mansit quadraginta dies secum. Et angelus ad Martinum dixit, ut iret Patricius ad Tamerensem insulam. " 
à Lérins reflètent l'attachement des Irlandais aux lieux et aux mouvements fondateurs qui furent à l'origine de leurs traditions monastiques.

En réaction à la propagande d'Armagh ou du moins à la même époque, le réseau d'Iona développe une stratégie similaire en faisant circuler le récit d'un séjour de Columba à Tours. Cette tradition est préservée dans une homélie en langue gaélique sur la vie de saint Columba :

Tan aile dó-sam i n-Doire iarum, \& nosimraid dula do Roim ocus do Ierusalem. Luid-sium fectus aile o Doire cu Torinis Martain co nus-tuc inso scéla boi for broinnib Mártain cét m-bliadan i talmain co nusfacaib i n-Doire*.

*Betha Coluim Chille, éd. Stokes, Whitley, Three Middle Irish Homilies on the lives of saints Patrick, Brigit and Columba, Calcutta, 1887, p. 90-124.
À une autre époque après cela, quand il résidait à Derry, il eut l'idée d'aller à Rome et à Jérusalem. Il alla une autre fois de Derry à Tours, [la ville] de Martin et ramena l'évangéliaire qui reposait sur la poitrine de Martin depuis cent ans sous terre et il le déposa à Derry.

Cette tradition reposait sur une réalité matérielle : la présence à Derry d'un évangéliaire, probablement originaire de Tours ou du moins du continent, considéré comme une relique martinienne par la communauté d'Iona. Deux récits différents, mais tout aussi fictifs, circulaient à son sujet. Le premier est que cet évangéliaire avait été légué à Columba par Patrick au moment de sa mort et que ce manuscrit était parvenu à Columba soit par l'intermédiaire de sainte Brigitte soit par celui d'un ange ${ }^{17}$. Le second est que saint Martin lui-même aurait légué son évangéliaire à Columba en commandant à ses proches d'enterrer le livre avec lui pour qu'il soit préservé intact jusqu'à l'arrivée de Columba cent ans plus tard. Le manuscrit était connu en Irlande médiévale sous le nom de Soiscel Martain "L'évangéliaire de Martin ». Nous savons par les Annales d'Ulster qu'il était encore à Derry en la possession du successeur de Columba en 1166, mais qu'il fut dérobé par les hommes de Jean de Courci à l'issue de la défaite qu'il infligea aux Cenél Eoghain en 1182 :

17. Manus O'Donnell, Betha Coluim Cille (éd. O'KeLLEHER Andrew et SCHOEPPERLE Gertrude, Betha Colaim Chille. Life of Columcille, Chicago, University of Illinois, 1918) ch. 28. 
Annales d'Ulster, AD1166

Rí Erenn do thuitim cen cath, cen cliathadh, iar sarughudh dó comarba Patraic \& Bachlu Isu \& comarba Coluim Cille \& Soscelai Martain \& cléirech ímda aile.

\section{$A D 1182$}

Sluagadh la Domnall h-Ua Lochlainn co Dun m-Bó i n-Dal Riatai \& cath do tabairt dó do Ghallaibh annsein \& maidm for Cenel $n$-Eogain \& Raghnall O Breislen do marbadh ann \& Gilla Crist $O$ Cathan do marbadh ann et alii multi. Ocus Soscela Martain do brith do Ghallaibh leó*

*Annales d'Ulster (éd. Mac Airt, Seán et Mac Niocaill, Gearóid, The Annals of Ulster (to AD 1131), Dublin, D.I.A.S., 1983), AD 1166 et 1182.
Le roi d'Irlande mourut sans bataille, sans combat après avoir déshonoré le successeur de Patrick et son bâton de Jésus, le successeur de Columba et son Évangéliaire de Martin et d'autres clercs autour d'eux.

Expédition militaire de Domnall Ó Lochlain à Dún mBó en Dál Ríata et il livra bataille aux Étrangers, mais les Cenél nEogáin furent battus et Raghnall Ó Breslein fut tué ainsi que Gilla Crist Ó Catháin et de nombreux autres. Les Étrangers emportèrent l'évangéliaire de Martin avec eux.

Au début du XVI ${ }^{\mathrm{e}}$ siècle, Manus O’Donnell rapporte une autre tradition qui lie l'acquisition de cet évangéliaire à l'élévation des reliques de Martin par Columba lors de son passage à Tours en revenant de Rome. Mais il semble que Manus O'Donnell ait attribué à Columba d'Iona la légende d'un autre saint du $\mathrm{VI}^{\mathrm{e}}$ siècle fondateur du monastère de Terryglass, nommé aussi Columba, mais plus connu sous le nom de Colmán - à moins, que le transfert n'ait eu lieu dans le sens contraire. En effet, la Vie de ce saint (Vita Columbae Tire Da Glas), qui date de la seconde moitié du VIII ${ }^{\mathrm{e}}$ siècle, relate l'expédition de Colmán à Rome pour en rapporter des reliques et son passage à Tours pour prier sur la tombe de saint Martin :

Cuius aduentum sanctus Martinus longe ante predixerat. Nam cum Martinus prope mortem infirmatus esset, dixit populo suo : "Ecce post obitum meum veniet ad uos quidam hospes sanctus de Hybernia insula, non ignobili genere, cum tali numero comitum et in tali habitu. Hic autem hospes meas reliquias de sepulcro eleuabit in tempore congruo. Ponite ergo istud crismale et trabeam iuxta me in scrinio, quia hec uexilla hospes ille a uobis postulabit, et dabitis illi* ${ }^{*}$,

${ }^{*}$ Vita S. Columbae Tire Da Glas (éd. Heist, William Watts, Vitae sanctorum Hiberniae, Bruxelles, Société des Bollandistes, 1965, p. 225-233) § 7.
Saint Martin avait prophétisé sa venue longtemps auparavant. En effet, alors que Martin était sans forces, proche de la mort, il dit à son peuple : "Voici qu'après ma mort arrivera chez vous un saint hôte de l'île d'Irlande, de famille noble, accompagné d'une suite éminente et vêtu d'une belle tenue. Et cet hôte élèvera mes reliques de mon tombeau en temps opportun. Posez donc cette pyxide et le manteau à mon côté dans mon tombeau, parce que l'hôte vous demandera ces insignes et vous les lui donnerez.

Bref, la prophétie de Martin se réalise. Colmán accomplit quelques miracles à Tours et procède à l'élévation du corps du saint, dont il lave les os et les dépose dans un reliquaire d'or et d'argent, puis repart avec la pyxide et le manteau. Qu'elles aient été authentiques ou non, des reliques martiniennes étaient vénérées à Terryglass au IX ${ }^{\mathrm{e}}$ siècle et sont mentionnées dans plusieurs autres textes. On aura remarqué de nouveau le motif du passage à 
Tours en route pour Rome ou sur le chemin du retour. Les mentions du pèlerinage à Tours dans ce contexte sont trop fréquentes pour être fortuites et reflètent certainement une réalité.

Cette vie de Colmán de Terryglass appartient à un troisième réseau martinien, celui des Céli Dé (Tallaght, Lorrha, Terryglass). Longtemps décrit comme un ordre monastique, ce mouvement, qui prend naissance dans le second quart du VIII ${ }^{\mathrm{e}}$ siècle, est en fait lié au renouveau de l'ascétisme dans certains monastères. Il s'est exprimé de différentes façons selon les personnes et les lieux, soit sous la forme d'un groupe d'ascètes résidant à l'extérieur de l'enceinte d'un monastère existant (comme à Roscrea) ou même à l'intérieur du monastère (comme à Armagh), soit sous la forme de nouveaux monastères indépendants, comme à Tallaght, fondé par Máelruain à la fin des années $760^{18}$. Le Céli Dé - dont le nom reprend la terminologie juridique séculière et signifie littéralement " client de Dieu " - se proclame vassal direct de Dieu et se distingue des autres moines par des obligations plus exigeantes envers son Seigneur, en matière de jeûnes, prières et mortifications. Le mouvement s'inspire du monachisme ascétique original que nous avons pu observer dans la Vita Columbae d'Adomnán - dont les modèles sont Antoine et Martin. C'est de ce milieu que sont issus deux martyrologes, fondés sur un exemplaire du Martyrologe hiéronymien utilisé à Iona, dans lequel Martin tient une place de choix. Rédigés entre 790 et 840 , le Martyrologe de Tallaght et le Félire Oengusso célèbrent saint Martin non seulement le 11 novembre - où le saint est qualifié de « mont d'or du monde occidental ${ }^{19}$ " - mais aussi le 4 juillet pour son ordination (" la belle et grande ordination de Martin $^{20} »$ ), le 4 juin pour la translation de ses reliques et, curieusement, le 20 avril où, de tous les saints européens, seul Martin reçoit une mention spéciale. Pour cette date, au texte irlandais du Félire Oengusso ("Fête à Rome, noble ville, des saints de toute l'Europe ${ }^{21}$ ") correspond le texte latin du Martyrologe de Tallaght : "Fête commune de tous les saints et vierges d'Irlande, de Bretagne et de toute l'Europe, et spécialement en l'honneur de saint Martin, évêque ${ }^{22}$. " Cette image de Martin, à la tête de la troupe des saints de l'Occident est reprise dans l'épilogue en vers du Félire Oengusso.

18. Voir Follett, Westley, Céli Dé in Ireland. Monastic writing and identity in the early Middle Ages, Woodbridge, Boydell Press, 2006.

19. Félire Oengusso (éd. STOKEs, Whitley, The martyrology of Oengus the Culdee, Londres, Henry Bradshaw Society, 1905) p. 234 : « iii. idus Novembris // Sanct Martain, sóer samuil, // slíab óir íarthair domuin ".

20. Ibidem, p. 160 : «iv. nonas Iulii. Dag-ordan már Martain ».

21. Ibid., p. 107 : "xii. Cal. Maii // Féil ir-Rúaim, rán baile // Nóeb n-Eoropa uile ".

22. Martyrologe de Tallaght (éd. BEST, Richard Irvine, et LAWLOR, Hugh Jackson, The Martyrology of Tallaght, Londres, Harrison and Sons, 1931) p. 34 : "xii. Cal. Maii // Communis solemnitas omnium sanctorum et uirginum Hiberniae et Britanniae et totius Europae et specialiter in honorem sancti Martini episcopi ". 
C'est aussi à Tallaght qu'est rédigé vers 800 le missel de Lorrha, qui contient la Deprecatio sancti Martini ${ }^{23}$. Le monastère de Lorrha est tout proche de Terryglass et c'est là que fut compilé le Leabhar Breac « Le Livre tacheté ", un recueil de textes qui comprend le Félire Oengusso, l'homélie sur la Vie de Columba d'Iona que nous avons citée plus haut, le Fís Adomnáin "La Vision d'Adomnán " et aussi une homélie en langue gaélique sur saint Martin. Écrite pour être prononcée le 11 novembre, cette homélie développe le thème de l'Évangile de Matthieu « Nul ne peut servir deux maîtres" (Matth. 6, 24) et cite l'exemple de Martin, modèle du choix du service divin contre le service impérial. Le reste de l'homélie est une adaptation très libre de la Vita Martini et des Dialogues de Sulpice Sévère. Elle se termine par un rappel de l'importance de ses reliques :

Atatt a thassi intii noem Mártain isna talmandaib co nglóir, co fertaib, co mírbulib, co nadamraib. Aanimm immurro taitnid isin catraig nemdai interceteros fideles Dei inter sidera*

De Virtute Sancti Mártain (éd. Stokes, Whitley, " A Middle-Irish Homily on S. Martin of Tours", Revue Celtique, $n^{\circ} 2,1875$, p. 381-402) $§ 44$.
Les reliques de saint Martin sont sur terre, avec leur gloire, avec leurs pouvoirs miraculeux, avec leurs merveilles, avec leurs grands prodiges. Mais son âme brille dans la cité céleste, au sein des autres fidèles de Dieu parmi les étoiles.

Cet ensemble de textes forme un contexte et atteste la grande vénération pour saint Martin dans l'Irlande du haut Moyen Âge. Conscients de leur position en marge de l'Europe, les Irlandais redoublent d'efforts pour affirmer leur orthodoxie et il est significatif que les grandes organisations monastiques que sont Iona et Armagh aient voulu être associées non seulement à Rome, mais aussi à Tours. Dès le vil ${ }^{\mathrm{e}}$ siècle, les pèlerinages locaux revêtent une grande importance pour le statut - mais aussi pour les finances - des centres ecclésiastiques irlandais et la possession de reliques romaines et martiniennes fait l'objet d'une forte compétition entre organisations rivales ${ }^{24}$. Le choix de toponymes tels que Disert Martain "L'ermitage de Martin », Temple Martin ou Kilmartin "L'église de Martin » dans les territoires associés aux trois réseaux que nous venons d'évoquer, tout autant que la fréquence de prénoms tels que Gilmartin "serviteur de Martin " ou Máelmartin " oblat de Martin ", confirme la permanence d'un culte encore très fort à l'arrivée des Anglo-Normands à la fin du XII ${ }^{\mathrm{e}}$ siècle. Ces derniers étaient conscients des enjeux politico-religieux, et lors de sa conquête de l'Irlande du Nord en 1177-1182, Jean de Courci s'empare non seulement des reliques des trois grands saints patrons de l'Irlande, Patrick, Brigitte

23. Voir plus haut p. 3 et note 8 .

24. Voir PICARD, Jean-Michel, "Le culte des reliques en Irlande (VII ${ }^{\mathrm{e}}-\mathrm{IX}{ }^{\mathrm{e}}$ siècles) ", dans BozóKy, Edina et Helvétius, Anne-Marie (dir.), Les Reliques. Objets, cultes, symboles, Turnhout, Brepols, 1999, p. 39-55. 
et Columba, mais aussi de la célèbre relique martinienne de Derry ${ }^{25}$. Aux $\mathrm{XIII}^{\mathrm{e}}$ et $\mathrm{XIV}^{\mathrm{e}}$ siècles, l'expansion de la présence anglo-française change les données de l'histoire du culte de saint Martin en Irlande. Les manifestations de la vénération pour saint Martin n'appartiennent plus uniquement au monde irlandais, mais s'inscrivent alors dans la culture religieuse du vaste empire Plantagenêt.

25. Sur la carrière de Jean de Courci en Irlande, voir Flanders, Steve, De Courcy. AngloNormans in Ireland, England and France in the eleventh and twelfth centuries, Dublin, Four Courts Press, 2008, p. 127-165. 